\title{
Toxicidad pulmonar por hiperoxia
}

\author{
F. Gordo-Vidal*, E. Calvo-Herranz, A. Abella-Álvarez e I. Salinas-Gabiña
}

Servicio de Medicina Intensiva, Hospital del Henares, Coslada, Madrid, España

Recibido el 14 de abril de 2009; aceptado el 20 de abril de 2009

Disponible en Internet el 24 de septiembre de 2009

\section{PALABRAS CLAVE \\ Hiperoxia; \\ Daño pulmonar; \\ Ventilación mecánica; \\ Efectos adversos; \\ Complicaciones}

\section{KEYWORDS}

Hyperoxia;

Lung injury;

Artificial respiration;

Adverse effects;

Complications

\begin{abstract}
Resumen
La ventilación mecánica es capaz de producir y agravar el daño pulmonar y contribuir a la aparición de fracaso multiorgánico. Uno de los mecanismos descritos es la hiperoxia alveolar que, en modelos experimentales, conlleva una producción de radicales libres de oxígeno $\left(\mathrm{O}_{2}\right)$ que exceden las posibilidades de defensa celular, y dan lugar a inflamación, a sobreexpresión genética y a daño celular directo con fenómenos de necrosis y apoptosis. Los hallazgos en humanos no son tan concluyentes, sí está claramente demostrada una alteración funcional debida a la exposición a la fracción inspiratoria de $\mathrm{O}_{2}\left(\mathrm{FiO}_{2}\right)$ elevada y a un mayor desreclutamiento pulmonar en los pacientes con lesión pulmonar, y que tanto la $\mathrm{FiO}_{2}$ empleada como la presión arterial de oxígeno conseguida en las primeras $24 \mathrm{~h}$ de ingreso están relacionadas con la mortalidad. Sería necesario realizar ensayos clínicos que evalúen cuál es el umbral de la $\mathrm{FiO}_{2}$ y de la saturación de $\mathrm{O}_{2}$ seguro.

(c) 2009 Elsevier España, S.L. y SEMICYUC. Todos los derechos reservados.
\end{abstract}

\section{Hyperoxia induced pulmonary toxicity}

\begin{abstract}
Mechanical ventilation may cause and aggravate lung damage and contribute to the appearance of multiorgan failure. One of the mechanisms that has been described is alveolar hyperoxia. In experimental models, it has lead to the production of free oxygen radicals that exceed the cell defense capacity, giving rise to inflammation, cell damage and gene overexpression with necrosis and apoptosis phenomenon. However, these findings in humans are not as conclusive, although a functional alteration due to the exposure to high $\mathrm{FiO}_{2}$, and greater lung de-recruitment in patients with lung injury has been clearly demonstrated. Moreover, both the $\mathrm{FiO}_{2}$ used as well as the $\mathrm{PaO}_{2}$ achieved in the first $24 \mathrm{~h}$ of admission are associated with mortality. Clinical trials are needed that assess the threshold of the safe oxygen level for $\mathrm{FiO}_{2}$ and oxygen saturation.

(c) 2009 Elsevier España, S.L. and SEMICYUC. All rights reserved.
\end{abstract}

\footnotetext{
*Autor para correspondencia.

Correo electrónico: fgordo5@gmail.com (F. Gordo-Vidal).
} 
Quizás, en el tratamiento de los pacientes en situación de insuficiencia respiratoria, la variación más importante que se ha producido en los últimos años ha sido el cambio en el concepto de que el propio soporte ventilatorio, aunque es evidentemente capaz de salvar vidas, también en determinadas circunstancias es capaz de producir daño pulmonar (sobre todo en pulmones previamente dañados) y condicionar la aparición de lesiones en otros órganos y sistemas que puedan contribuir con la aparición del fracaso múltiple orgánico $^{1-3}$. Este cambio de concepto explicaría, en parte, el porqué la mayoría de los pacientes con lesión pulmonar aguda (LPA) no fallecen en situación de hipoxemia refractaria $^{4}$, sino en situación de disfunción múltiple orgánica ${ }^{5,6}$. Se han descrito diferentes mecanismos por los que se produce esta lesión pulmonar ${ }^{7,8}$ : daño pulmonar inducido por presión excesiva en la vía aérea (barotrauma) y sobredistensión pulmonar (volutrauma), daño pulmonar inducido por bajo volumen total (atelectrauma), daño por liberación de mediadores (biotrauma) y daño pulmonar producido por el empleo de fracciones inspiratorias de oxígeno $\left(\mathrm{O}_{2}\right)$ elevadas (hiperoxia). Todos estos mecanismos han sido demostrados en estudios experimentales, tanto en cultivos celulares como en modelos in vivo en animales, y alguno de estos mecanismos ha sido demostrado e incluso valorado su efecto y posibles alternativas terapéuticas en humanos ${ }^{9-12}$.

Ya en la década de 1960 se describió la exposición a las fracciones inspiratorias de $\mathrm{O}_{2}\left(\mathrm{FiO}_{2}\right)$ elevadas, en ventilación mecánica, como productora de alteraciones del intercambio gaseoso y como posible productora de alteraciones estructurales del aparato respiratorio y específicamente del parénquima pulmonar ${ }^{13}$. Entre los mecanismos claramente demostrados para la aparición de estas alteraciones funcionales en humanos están fundamentalmente los "mecánicos", consistentes en la producción de atelectasias por reabsorción, la hipercapnia hiperóxica, el daño del epitelio bronquial con aparición de bronquitis hiperóxica y disminución de la eficacia del epitelio ciliar y de la función bactericida bronquial ${ }^{14}$. Obviamente, todos estos fenómenos dan lugar a una alteración funcional, consistente en la disminución de la distensibilidad pulmonar, con la consiguiente alteración en la mecánica pulmonar y empeoramiento de la capacidad de intercambio gaseoso ${ }^{15}$, además de un aumento de la susceptibilidad a la infección.

Sin embargo, existe controversia en cuanto a si el $\mathrm{O}_{2}$ es capaz de inducir una lesión pulmonar por daño celular directo alveolar en humanos ("mecanismo biológico"). Desde la década de 1960 se empezó a mencionar que el empleo de oxigenoterapia prolongada puede producir lesiones similares al distrés respiratorio. A modo de ejemplo, en 1974 Sevitt $^{16}$ publicó un artículo en el que literalmente refería que "en pacientes que respiran concentraciones altas de oxígeno a presión atmosférica, se puede producir una alteración pulmonar grave. Se desarrollan opacidades radiológicas en ambos pulmones, aumenta la resistencia en la vía aérea y se produce hipoxemia, que aunque es reversible por un tiempo, a menudo se convierte refractaria incluso al empleo de oxígeno puro. Los pulmones se hepatizan y muestran membranas hialinas, y con una mayor supervivencia una pneumonitis proliferativa que afecta al epitelio alveolar y los fibroblastos. Se ha sugerido la ventilación a presión positiva como una posible causa de esta afectación pulmonar se ha descartado mediante experimentos en cabras, y la conclusión de que la toxicidad por oxigeno es la causa de esta lesión no puede ser descartada". Con esta hipótesis hace un estudio histológico de los pulmones de 21 pacientes que fallecieron tras un tratamiento con $\mathrm{O}_{2}$, la mayoría de los pacientes había tenido lesiones que ahora sabemos que son factores de riesgo para el desarrollo de una LPA (traumatismo torácico, traumatismo ortopédico, politraumatismo e inhalación de humo en pacientes quemados) $y$, de éstos, prácticamente todos precisaron ventilación mecánica durante un período prolongado. Los resultados del estudio fueron que prácticamente todos los pacientes que precisaron $\mathrm{FiO}_{2}$ superior al $50 \%$ presentaron una pneumonitis difusa con membranas hialinas, mientras que los que precisaron una $\mathrm{FiO}_{2}$ inferior presentaron pneumonitis focal. Éste y otros artículos similares publicados en la década de 1970 generaron la hipótesis de que el empleo de una $\mathrm{FiO}_{2}$ superior al $50 \%$ en los pacientes que precisaban soporte ventilatorio, por cualquier motivo, podía inducir una lesión pulmonar compatible con un síndrome de distrés respiratorio agudo por un daño celular directo sobre las células alveolares y no sólo una alteración funcional reversible condicionada por una disminución del volumen pulmonar que fue claramente demostrado en enfermos que recibieron cirugía prolonga$\mathrm{da}^{17}$. Estos artículos condicionaron la recomendación generalizada de que el empleo de oxigenoterapia con $\mathrm{FiO}_{2}$ superiores al 50\% produce lesión pulmonar directa. Desde entonces, se ha progresado enormemente en el conocimiento de los mecanismos fisiológicos de la LPA y de sus factores de riesgo y predisponentes.

En el momento actual, sin embargo, gracias a múltiples estudios, conocemos que el empleo de ${ }^{18}$ una $\mathrm{FiO}_{2}$ elevada sí puede inducir un efecto lesivo directo sobre las células del parénquima pulmonar. Estos efectos se pueden resumir en las siguientes líneas generales:

La exposición del pulmón a niveles altos de $\mathrm{O}_{2}$ da lugar a la producción de derivados, tales como el anión superóxido, el peróxido de hidrógeno y radicales hidroxilo, estas sustancias actúan sobre una superficie pulmonar muy amplia y con una importante vascularización, lo que convierte al parénquima pulmonar en muy susceptible al efecto tóxico de estas sustancias (estrés oxidativo). El efecto tóxico se produce cuando la producción de estos derivados excede la capacidad de los mecanismos de defensa antioxidante ${ }^{19}$. El mayor productor de estos derivados de $\mathrm{O}_{2}$ es el sistema NADPH oxidasa y la mitocondria 20,21 .

También conocemos que al modular enzimas antioxidantes podemos inducir una protección frente al efecto tóxico de los radicales libres de $\mathrm{O}_{2}$. Así, en ratones transgénicos, Wang et $\mathrm{al}^{22}$ han demostrado cómo la sobreexpresión de un agente antioxidante (peroxiredoxin 6) es capaz de reducir la peroxidación lipídica y el daño pulmonar inducido por hiperoxia. Sin embargo, otros modelos experimentales similares en los que se sobreexpresan otros agentes antioxidantes han tenido resultados negativos ${ }^{23}$. A modo de ejemplo, en animales a los que se induce una lesión pulmonar impulsada por ventilación mecánica tratados con melatonina, en busca de su efecto antioxidante, se demostró cómo en una situación de hiperoxia $\left(\mathrm{O}_{2}\right.$ al $\left.50 \%\right)$ la melatonina tenía un efecto paradójico prooxidante ${ }^{24}$. Éste es un efecto secundario de prácticamente todos los 
antioxidantes, que en situaciones adversas pueden tener efectos opuestos por su naturaleza química.

En modelos animales, la hiperoxia prolongada (7 días con $\mathrm{FiO}_{2}$ al 100\%) da lugar a alteraciones histopatológicas similares a las encontradas en la LPA o en los modelos de lesión pulmonar inducida por el respirador. Estas alteraciones incluyen la aparición de edema, formación de membranas hialinas, lesiones vasculares y fibrosis pulmonar ${ }^{13,25}$. La hiperoxia alveolar mantenida da lugar a fenómenos de destrucción celular, que se han podido observar tanto en modelos de cultivos celulares como in vivo ${ }^{26,27}$. Estos fenómenos se producen por diferentes vías, tanto por necrosis como por apoptosis ${ }^{23}$. Los signos más precoces de daño celular por hiperoxia, en modelos experimentales en ratas, aparecen en las células endoteliales de los capilares pulmonares que muestran signos de necrosis consistentes en rotura de las membranas citoplásmicas y nucleares y destrucción de organelas ${ }^{28-30}$.

Recientemente, se está acumulando evidencia que demuestra un papel fundamental de algunos mediadores en el desarrollo del daño celular inducido por hiperoxia o por los radicales libres de $\mathrm{O}_{2}$. Se ha observado un incremento de la actividad de la caspasa en diferentes modelos de pulmones hiperóxidos (la activación de la caspasa se considera como una vía irreversible de la apoptosis) ${ }^{31}$. Así, se ha demostrado un papel crítico de la activación de la caspasa 3 en el desarrollo de esta muerte celular ${ }^{27}$. El estrés oxidativo puede inducir apoptosis en un período de tiempo relativamente corto (horas).

Otros efectos de la hiperoxia son la inducción de una expresión aumentada en la producción de diferentes citoquinas y factores de crecimiento vascular endotelial y la activación de la matriz extracelular, lo que tiene profundas implicaciones en la lesión y reparación pulmonar en diferentes modelos ${ }^{30,32-34}$.

Aunque, como hemos visto, se han desarrollado muy diferentes modelos experimentales para estudiar el efecto de la hiperoxia sobre el pulmón, sin embargo y paradójicamente se han publicado pocos artículos que hagan referencia a la interacción sobre el pulmón entre la hiperoxia y el soporte ventilatorio. Bailey et $\mathrm{al}^{35}$ han descrito cómo los pulmones de ratón expuestos a $\mathrm{O}_{2}$ al $90 \%$ durante $48 \mathrm{~h}$ fueron más susceptibles al daño pulmonar inducido por la ventilación mecánica que los pulmones expuestos previamente a aire ambiente. En un modelo de conejos ventilados mecánicamente con volumen de $25 \mathrm{ml} / \mathrm{kg}$ y presión positiva al final de la espiración (PEEP) de $0 \mathrm{~cm}$ de $\mathrm{H}_{2} \mathrm{O}$, Sinclair et al encontraron que la hiperoxia incrementa el daño pulmonar inducido en el modelo de volutrauma ${ }^{36}$.

Este pequeño resumen de los hallazgos experimentales del efecto tóxico directo sobre las células pulmonares hace referencia en todo momento a hallazgos sobre los pulmones de animales de pequeño tamaño, en los modelos experimentales y en los cultivos celulares. Los datos de estas investigaciones parecen indicar que la producción de radicales libres de $\mathrm{O}_{2}$ en una cantidad que excede las posibilidades de defensa antioxidante de las células pulmonares da lugar a la liberación de mediadores, sobreexpresión genética y daño celular directo con fenómenos de necrosis mitocondrial y apoptosis celular.

¿Qué se produce en humanos o en la práctica clínica? Pues por el momento no hay ninguna evidencia de la producción de este daño directo sobre las células inducido por la necesidad de emplear niveles altos de $\mathrm{O}_{2}$. En humanos sanos, la exposición a $\mathrm{O}_{2}$ al $100 \%$ produce una progresiva reducción en la capacidad vital forzada y en la capacidad residual funcional, atelectasias, alteración del aclaramiento mucociliar y traqueobronquitis ${ }^{14}$. En pacientes con LPA, Aboab et $\mathrm{al}^{37}$ estudiaron 40 pacientes con distintos niveles de $\mathrm{FiO}_{2}$ (al 100 y al 60\%) con diferentes niveles de PEEP y describieron cómo en pacientes con soporte ventilatorio el empleo de la $\mathrm{FiO}_{2}$ al $100 \%$ produjo un mayor colapso o desreclutamiento pulmonar que, sin embargo, es fácilmente prevenible con el empleo de PEEP.

En todo caso, lo que sí está claramente demostrado es el efecto nocivo de la hipoxia sobre los tejidos periféricos. Se ha intentado emplear la hiperoxia como arma terapéutica, sin embargo, también pocos ensayos clínicos han evaluado su efecto. Se ha indicado que la hiperoxia normobárica puede mejorar el pronóstico en los pacientes con accidentes cerebrovasculares isquémicos ${ }^{38}$ y existe controversia en si la hiperoxia puede reducir las tasas de infección en pacientes quirúrgicos de alto riesgo ${ }^{39-41}$.

Recientemente, De Jonge et $\mathrm{al}^{42}$ han publicado un análisis retrospectivo de los datos de oxigenación de las primeras $24 \mathrm{~h}$ de ingreso de 36.307 pacientes ingresados en $50 \mathrm{UCl}$ y ventilados mecánicamente, con el objetivo de describir los objetivos de oxigenación empleados en éstos y establecer si el pronóstico de los pacientes se asoció a diferencias en la $\mathrm{FiO}_{2}$ administrada a éstos o a diferencias en la $\mathrm{PaO}_{2}$ conseguida. Los autores describen cómo la $\mathrm{FiO}_{2}$ empleada en las primeras $24 \mathrm{~h}$ de ingreso muestra una relación lineal con la mortalidad, mientras que la $\mathrm{PaO}_{2}$ conseguida se asocia con un incremento de la mortalidad, tanto en los valores más bajos como en los más altos. Estos hallazgos son independientes del resto de las variables incluidas en un análisis multivariable (SAPS II, tipo de ingreso, Glasgow coma score, y $\mathrm{PaO}_{2} / \mathrm{F}_{\mathrm{i}} \mathrm{O}_{2}$ en el ingreso y en el hospital). La conclusión de los autores a sus resultados es que la administración de $\mathrm{O}_{2}$ en sí misma puede tener un efecto perjudicial, y que la asociación entre la $\mathrm{FiO}_{2}$ empleada y la mortalidad no puede explicarse mediante otros factores de confusión. Sin embargo, el propio diseño retrospectivo de este estudio plantea serias dudas sobre sus resultados y sobre el efecto de la $\mathrm{FiO}_{2}$ en este supuesto aumento de la mortalidad.

A pesar de los numerosos avances en el conocimiento de los posibles factores que mediarían el daño pulmonar inducido por hiperoxia alveolar, es necesario profundizar en su real aplicación en la práctica clínica. En el momento actual quizás podamos establecer que:

1. El empleo de $\mathrm{FiO}_{2}$ elevada puede producir un efecto tóxico directo sobre las células pulmonares, con fenómenos de destrucción celular (tanto por apoptosis como por muerte celular inducida a causa de la mitocondria) y alteración de los mecanismos de defensa; todos estos fenómenos inducidos por la sobreproducción de radicales libres de $\mathrm{O}_{2}$. Estos fenómenos han sido demostrados en cultivos celulares y modelos experimentales en animales de pequeño tamaño sometidos a hiperoxia prolongada. En humanos se puede establecer que el empleo de $\mathrm{FiO}_{2}$ elevada puede producir un ambiente oxidante local que 
puede agravar la lesión pulmonar inducida por el respirador.

2. El empleo de $\mathrm{FiO}_{2}$ elevada en humanos produce atelectasias por reabsorción, la hipercapnia hiperóxica, daño del epitelio bronquial con aparición de bronquitis hiperóxica y disminución de la eficacia del epitelio ciliar y de la función bactericida bronquial. Obviamente, todos estos fenómenos dan lugar a una alteración funcional, consistente en disminución de la distensibilidad pulmonar, con la consiguiente alteración en la mecánica pulmonar y empeoramiento de la capacidad de intercambio gaseoso. En ventilación mecánica la $\mathrm{FiO}_{2}$ elevada además condiciona un desreclutamiento pulmonar, aunque este efecto es fácilmente prevenible con el empleo de PEEP.

3. No hay efectos beneficiosos de la hiperoxia en pacientes críticos claramente demostrados en ensayos clínicos.

4. Lo que sí está claramente demostrado es el efecto nocivo de la hipoxia sobre los tejidos periféricos.

Quizás la evidencia disponible en este momento nos obligue a ser cautos en la administración de $\mathrm{O}_{2}$ en pacientes con ventilación mecánica, especialmente en aquéllos con LPA, aunque como en casi todos los campos de la medicina no deberíamos ser dogmáticos en el paradigma de que la oxigenoterapia puede inducir daño, ya que lo que sí está claramente demostrado es el efecto perjudicial de la hipoxemia. Es posiblemente necesario desarrollar ensayos clínicos que testen cuál es realmente el umbral de la $\mathrm{FiO}_{2}$ y de la saturación de $\mathrm{O}_{2}$ seguro en nuestros pacientes.

\section{Bibliografía}

1. Gordo F, Delgado C, Calvo E. Lesión pulmonar inducida por la ventilación mecánica. Med Intensiva. 2007;31:18-26.

2. Parker JC, Hernández LA, Peevy KJ. Mechanisms of ventilatorinduced lung injury. Crit Care Med. 1993;21:131-43.

3. Tremblay LN, Slutsky AS. Ventilator-induced injury: From barotrauma to biotrauma. Proc Assoc Am Physicians. 1998;110: 482-488.

4. Esteban A, Fernández-Segoviano P, Frutos-Vivar F, Aramburu JA, Najera L, Ferguson ND, et al. Comparison of clinical criteria for the acute respiratory distress syndrome with autopsy findings. Ann Intern Med. 2004;141:440-5.

5. Slutsky AS, Tremblay LN. Multiple system organ failure. Is mechanical ventilation a contributing factor?. Am J Respir Crit Care Med. 1998;157:1721-5.

6. Offner PJ, Moore EE. Lung injury severity scoring in the era of lung protective mechanical ventilation: The $\mathrm{PaO}_{2} / \mathrm{FIO}_{2}$ ratio. $\mathrm{J}$ Trauma. 2003;55:285-9.

7. Marini JJ, Gattinoni L. Propagation prevention: A complementary mechanism for "lung protective" ventilation in acute respiratory distress syndrome. Crit Care Med. 2008;36:3252-8.

8. Tang PS, Mura M, Seth R, Liu M. Acute lung injury and cell death: How many ways can cells die?. Am J Physiol Lung Cell Mol Physiol. 2008;294:632-41.

9. Brower RG, Lanken PN, Maclntyre N, Matthay MA, Morris A, Ancukiewicz $M$, et al. Higher versus lower positive endexpiratory pressures in patients with the acute respiratory distress syndrome. N Engl J Med. 2004;351:327-36.

10. Brower RG, Morris A, Maclntyre N, Matthay MA, Hayden D, Thompson $\mathrm{T}$, et al. Effects of recruitment maneuvers in patients with acute lung injury and acute respiratory distress syndrome ventilated with high positive end-expiratory pressure. Crit Care Med. 2003;31:2592-7.

11. Ventilation with lower tidal volumes as compared with traditional tidal volumes for acute lung injury and the acute respiratory distress syndrome. The Acute Respiratory Distress Syndrome Network. N Engl J Med. 2000;342:1301-8.

12. Gordo-Vidal F, Gómez-Tello V, Palencia-Herrejon E, Latour-Pérez J, Sánchez-Artola B, Az-Alersi R. PEEP alta frente a PEEP convencional en el síndrome de distrés respiratorio agudo: revisión sistemática y metaanálisis. Med Intensiva. 2007;31:491-501.

13. Nash G, Blennerhassett JB, Pontoppidan H. Pulmonary lesions associated with oxygen therapy and artificial ventilation. $N$ Engl J Med. 1967;276:368-74.

14. Carvalho CR, De Paula Pinto SG, Maranhao B, Bethlem EP. Hyperoxia and lung disease. Curr Opin Pulm Med. 1998;4:300-4.

15. Fracica PJ, Knapp MJ, Piantadosi CA, Takeda K, Fulkerson WJ, Coleman RE, et al. Responses of baboons to prolonged hyperoxia: Physiology and qualitative pathology. J Appl Physiol. 1991;71:2352-62.

16. Sevitt S. Diffuse and focal oxygen pneumonitis. A preliminary report on the threshold of pulmonary oxygen toxicity in man. J Clin Pathol. 1974;27:21-30.

17. Hedenstierna G, Rothen HU. Atelectasis formation during anesthesia: Causes and measures to prevent it. J Clin Monit Comput. 2000;16:329-35.

18. Hayatdavoudi G, O’Neil JJ, Barry BE, Freeman BA, Crapo JD. Pulmonary injury in rats following continuous exposure to $60 \%$ $\mathrm{O}_{2}$ for 7 days. J Appl Physiol. 1981;51:1220-31.

19. Zaher TE, Miller EJ, Morrow DM, Javdan M, Mantell LL. Hyperoxia-induced signal transduction pathways in pulmonary epithelial cells. Free Radic Biol Med. 2007;42:897-908.

20. Freeman BA, Topolosky MK, Crapo JD. Hyperoxia increases oxygen radical production in rat lung homogenates. Arch Biochem Biophys. 1982;216:477-84.

21. Freeman BA, Crapo JD. Hyperoxia increases oxygen radical production in rat lungs and lung mitochondria. J Biol Chem. 1981;256:10986-92.

22. Wang $\mathrm{Y}$, Phelan SA, Manevich $\mathrm{Y}$, Feinstein SI, Fisher AB. Transgenic mice over expressing peroxiredoxin 6 show increased resistance to lung injury in hyperoxia. Am J Respir Cell Mol Biol. 2006;34:481-6.

23. Altemeier WA, Sinclair SE. Hyperoxia in the intensive care unit: Why more is not always better. Curr Opin Crit Care. 2007;13: 73-78.

24. Pedreira PR, García-Prieto E, Parra D, Astudillo A, Díaz E, Taboada F, et al. Effects of melatonin in an experimental model of ventilator-induced lung injury. Am J Physiol Lung Cell Mol Physiol. 2008;295:L820-7.

25. Jones R, Zapol WM, Reid L. Oxygen toxicity and restructuring of pulmonary arteries-a morphometric study. The response to 4 weeks' exposure to hyperoxia and return to breathing air. Am J Pathol. 1985;121:212-23.

26. Husari AW, Dbaibo GS, Bitar H, Khayat A, Panjarian S, Nasser M, et al. Apoptosis and the activity of ceramide, Bax and Bcl-2 in the lungs of neonatal rats exposed to limited and prolonged hyperoxia. Respir Res. 2006;7:100.

27. Wang X, Ryter SW, Dai C, Tang ZL, Watkins SC, Yin XM, et al. Necrotic cell death in response to oxidant stress involves the activation of the apoptogenic caspase-8/bid pathway. J Biol Chem. 2003;278:29184-91.

28. Kistler GS, Caldwell PR, Weibel ER. Development of fine structural damage to alveolar and capillary lining cells in oxygen-poisoned rat lungs. J Cell Biol. 1967;32:605-28.

29. Kistler GS, Weibel ER, Caldwell PR. Electron microscopic investigations of oxygen effects on lung tissue. AMRL-TR-66120. AMRL TR. 1966:108-61.

30. Crapo JD, Barry BE, Foscue HA, Shelburne J. Structural and biochemical changes in rat lungs occurring during exposures to 
lethal and adaptive doses of oxygen. Am Rev Respir Dis. 1980;122:123-43.

31. Pedreira PR, García-Prieto E, Albaiceta GM, Taboada F. Respuesta inflamatoria y apoptosis en la lesión pulmonar aguda. Med Intensiva. 2006;30:268-75.

32. Liu W, Hendren J, Qin XJ, Shen J, Liu KJ. Normobaric hyperoxia attenuates early blood-brain barrier disruption by inhibiting MMP-9-mediated occludin degradation in focal cerebral ischemia. J Neurochem. 2009;108:811-20.

33. Chetty A, Cao GJ, Severgnini M, Simon A, Warburton R, Nielsen $\mathrm{HC}$. Role of matrix metalloprotease- 9 in hyperoxic injury in developing lung. Am J Physiol Lung Cell Mol Physiol. 2008;295: L584-L592.

34. Fu JH, Xue XD. Gene expressions and roles of matrix metalloproteinases- 8 and tissue inhibitor of metalloproteinases-1 in hyperoxia-induced pulmonary fibrosis in neonatal rats. Zhongguo Dang Dai Er Ke Za Zhi. 2007;9:1-5.

35. Bailey TC, Martin EL, Zhao L, Veldhuizen RA. High oxygen concentrations predispose mouse lungs to the deleterious effects of high stretch ventilation. J Appl Physiol. 2003;94:975-82.

36. Sinclair SE, Altemeier WA, Matute-Bello G, Chi EY. Augmented lung injury due to interaction between hyperoxia and mechanical ventilation. Crit Care Med. 2004;32:2496-501.
37. Aboab J, Jonson B, Kouatchet A, Taille S, Niklason L, Brochard L. Effect of inspired oxygen fraction on alveolar derecruitment in acute respiratory distress syndrome. Intensive Care Med. 2006;32:1979-86.

38. Chiu EH, Liu CS, Tan TY, Chang KC. Venturi mask adjuvant oxygen therapy in severe acute ischemic stroke. Arch Neurol. 2006;63:741-4.

39. Pryor KO, Fahey III TJ, Lien CA, Goldstein PA. Surgical site infection and the routine use of perioperative hyperoxia in a general surgical population: A randomized controlled trial. JAMA. 2004;291:79-87.

40. Pryor KO, Lien CA, Fahey III TJ, Goldstein PA. Supplemental oxygen and risk of surgical wound infection. JAMA. 2006;295: 1642-1643.

41. Greif R, Akca O, Horn EP, Kurz A, Sessler DI, Outcomes Research Group. Supplemental perioperative oxygen to reduce the incidence of surgical-wound infection. N Engl J Med. 2000;342: 161-167.

42. De Jonge E, Peelen L, Keijzers PJ, Joore H, De Lange D, Van der Voort $\mathrm{PH}$, et al. Association between administered oxygen, arterial partial oxygen pressure and mortality in mechanically ventilated intensive care unit patients. Crit Care. 2008;12: R156. 